\title{
Optimization of the support structure of a multipurpose machine tool
}

\author{
Aleksandr Yagopolskiy $^{1}$, Dmitry Tutukin ${ }^{1}$, Nikolay Andryukhin ${ }^{1 *}$, and Dmitry Ivanov ${ }^{1}$ \\ ${ }^{1}$ Bauman Moscow State Technical University, ul. Baumanskaya 2-ya, 5, Moscow, Russia
}

\begin{abstract}
Modern manufacturers of multipurpose machining centers design and manufacture equipment according to the criterion of maximum rigidity and productivity to ensure processing of the maximum range of materials at high accelerated cutting modes. As a result, in most machinebuilding tasks, the support structure of the machine is unnecessarily rigid due to the production of excessively massive frames and body elements of the machine. These structural elements have high weight and size characteristics, which leads to an increase in inertial loads on the drive feed mechanisms. In serial and mass production, heavy elements of the support structure significantly increase the energy consumption of the machine, while reducing labor productivity. Modern methods of design optimization, together with finite element methods, allow solving the problem of energy efficiency and productivity by modifying the support structures according to the criterion of minimum rigidity in accordance with the boundary conditions of the cutting forces when processing the selected product range.
\end{abstract}

\section{Introduction}

When designing a product, modern manufacturers of metalworking machine tools strive to create an excessively rigid structure of support systems [1] in order to provide the ability to process a large range of parts without losing rigidity and accuracy. Especially productive tasks can create unnecessary stress and lead to increased deformations in the elastic system, which inevitably leads to a decrease in the nominal machining accuracy. The increased material consumption of the support structures of the machine tool and a large rigidity margin leads to a decrease in accuracy due to the increased inertia of the units [2], increases the power consumption of the machine tool due to the movement of greater masses. In modern mechanical engineering, there are design optimization methods that allow modernization of the existing geometry and reduction of material consumption while maintaining the specified accuracy.

\section{Materials and methods}

As a rule, the geometry of standard columns [3] of milling machine tools is a complex spatial system with many transverse and longitudinal stiffeners to prevent excessive

* Corresponding author: nikolay2198@,mail.ru 
allowable deformation. It is important to note that during the operation of the machine tool, the initial geometry of a hollow box column without stiffeners considered in this paper may not provide sufficient rigidity during the milling operation. In this work, the study of the existing geometry of the column was carried out according to the criterion of operability, and the permissible minimum wall thickness was found while maintaining the specified stiffness.

The column of a CNC milling machine tool was chosen as an experimental model for optimizing the bearing system of a multipurpose machine tool. Figure 1 shows the main components of the object under consideration: base 2, column 4, arm 6, milling head 7, guide rails 1 . The column design is a box-like structure with a small wall thickness $t=50$ $\mathrm{mm}$, without additional internal stiffeners.

The main methods for identifying and solving a given problem [4] are: topological and parametric optimization. As a method for solving the problem, the method of parametric optimization was chosen, since changing the topology has significant disadvantages [5], such as:

1) The output data does not give an unambiguous understanding of the operation of the structure over the entire load range and changes significantly when the load vector changes.

2) Topology analysis assumes significant design changes [6].

Parametric optimization is a method that allows analyzing the design of a structure by varying the input boundary parameters: geometric, force, contact, etc. [7]. In the general case, each set of values of the parameters of the system corresponds to a certain trajectory or a set of trajectories of the system - a field of behavior. This method has the following advantages:

1) Parametric optimization gives an unambiguous understanding of the change in the output parameters of the system in the full field of values.

2) Parametric control does not require changes to the original developed system.

3) Parametric optimization and control can be applied at all stages of design to assess quality criteria.

4) The possibility of obtaining the extremum of the parameter of interest.

In the process of optimization, the most rational research method is the use and variation of not all parameters at the same time, but only those that have a significant impact on changing the criteria of interest, in particular, design elements of the structure [8]. Obtaining the necessary data assumes the presence of a mathematical model of the development object, which can unambiguously express the dependence of the output parameters $\mathrm{Y}$ when changing control objects - parameters $\mathrm{X}$. The simplest case is dependence (1):

$$
Y=F(X)
$$

where $F=\left(f_{1}, f_{2}, \ldots, f_{m}\right)$ may include functional and algorithmic dependencies. When written in scalar form, the formula is a matrix of values:

$$
\begin{aligned}
y_{1} & =f_{1}\left(x_{1}, x_{2}, \ldots, x_{n}\right) \\
y_{2} & =f_{2}\left(x_{1}, x_{2}, \ldots, x_{n}\right) \\
y_{m} & =f_{m}\left(x_{1}, x_{2}, \ldots, x_{n}\right)
\end{aligned}
$$



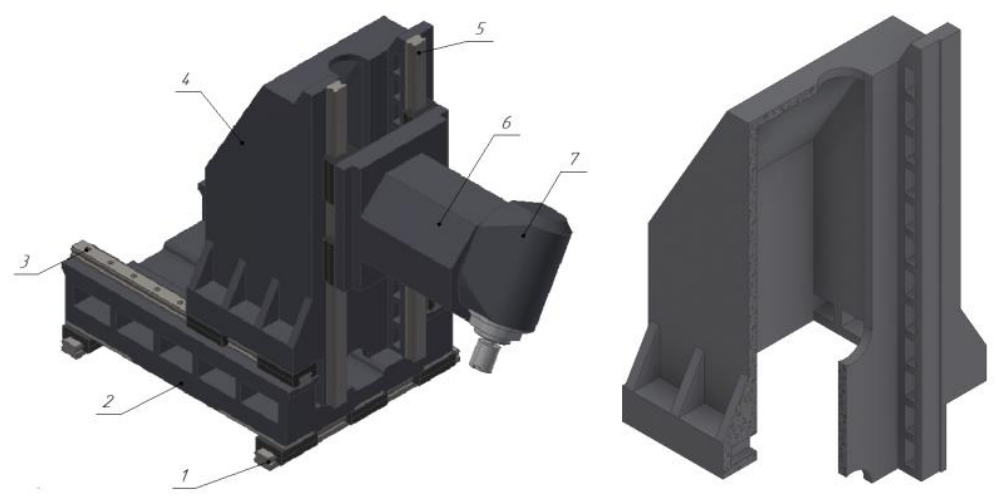

Fig. 1. Milling column of the machine.

Optimal design requires the compilation and selection of an objective function, which is repeated by cycles of analyzing the values of the output parameters, and as a consequence, the minimization or maximization of the objective function using various optimization methods, the choice of a particular one is caused by the specifics of the given problem being solved [9]. As a means of calculation, we will use the finite element method (FEM) and the calculation of the Inventor 3D CAD system.

\section{Results}

Within the framework of the study, geometry elements that did not significantly affect the results of the experiment were initially eliminated. For this, the initial unit was loaded with force factors exceeding the specified value, namely, the force $\mathrm{F}=10,000 \mathrm{~N}$, to the end face of the milling spindle. As an output parameter, the maximum elastic displacements in the body of the bearing system were selected, as a qualitative parameter for assessing the rigidity. The calculation result is shown in Fig. 2. It can be seen that during milling at loads exceeding the maximum by 2-3 times of the nominal, the maximum deformation (displacement) distribution fields reach their critical values only within the column. As a result, the remaining elements of the system were excluded from the study, since they do not significantly contribute to the results.

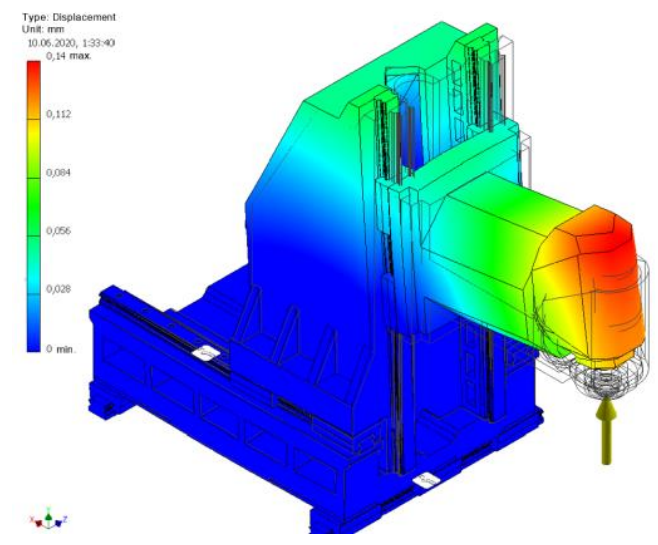

Fig. 2. Deformation distribution fields in the machine tool unit. 
The main parameter is the final machining accuracy of the part for ensuring the specified accuracy, which directly depends on the local displacements of the cutting tool vertices when exposed to the cutting force [10]. The overall rigidity of the entire system of the unit under consideration plays a significant role in changing the vertex position [11]. On this basis, to assess the final processing accuracy, a directly proportional dependent value is used - the rigidity of the system (2):

$$
j=F / \chi=[N / \mu m]
$$

where $\mathrm{F}$ - force acting on the system $[\mathrm{N}]$,

$\chi$ - displacement arising under the action of the force.

For high-precision machine tools, this indicator should be large $j_{\text {lim }} \geq$ $450 \ldots 500 \frac{N}{\mu \mathrm{m}}$; therefore, the average permissible value $j_{\text {lim }}=475 \frac{N}{\mu \mathrm{m}}$ [12].

As an input variable parameter, the column wall thickness $t$ was chosen, which directly proportionally affects the rigidity of the system. The total fields of the cutting force are considered in the range of maximum displacements of the unit along the vertical and rotary axes (see Fig. 3).

The amount of deformation of the unit elements is measured in accordance with the angular position of the point of application of forces (i.e. with the rotation of the milling head) around the A axis, and simultaneously with the change in the position along the vertical Y axis. The displacements from the impact of force factors were calculated in the working field of displacements of the operating device along the $\mathrm{Y}$ axis $=250 . .500 \mathrm{~mm}$, along the angular coordinate $\varphi=0 \ldots 360 \mathrm{deg}$.

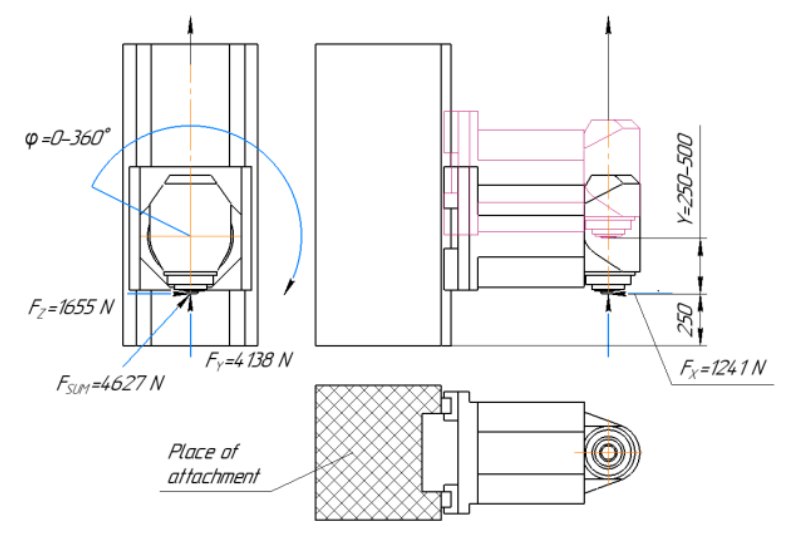

Fig. 3. Fixing scheme. Force application diagram. Measuring range.

To identify the maximum possible rigidity of the initial geometry, a study of the frame was carried out with full filling of the inner cavity of the column structure with material. Material - cast iron (Young's modulus $120.5 \mathrm{GPa}$, shear - $58100 \mathrm{MPa}$ ) [13]. The measurement results were obtained in 11 angular positions with a step of 30 degrees relative to the rotary axis and in 11 linear positions along the $\mathrm{Y}$ axis with a step of $25 \mathrm{~mm}$ (see Fig. 4): 


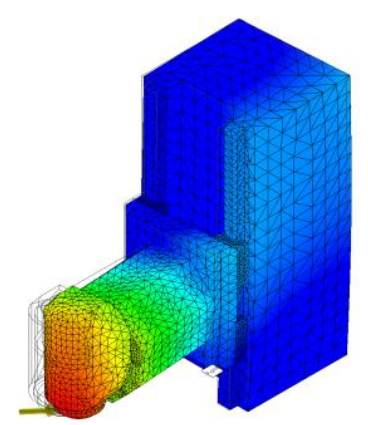

Fig. 4. FE study model.

As a result of varying two input parameters and obtaining one output parameter, a threedimensional plane of values was formed. Within the framework of the study, the scalar distribution surface $\chi=\chi(Y, \varphi)$ was analyzed, without taking into account the directions of displacements, proceeding only from the total global vector value of displacements in the elastic system and its gradient $\psi=\operatorname{grad}(\chi)$ (its projection onto the $\mathrm{Y} \varphi$ plane) in figure 5.

From the gradient of the scalar field, an unambiguous conclusion was made about the distribution area of the maximum displacements in the structure. The maximum deformation occurs in the area at $Y=420-500, \varphi=100-200$. The function of the dependence of shifts on displacements along $\mathrm{Y} \chi \mathrm{Y}=\chi \mathrm{Y}(\mathrm{Y})$ increases monotonically in the definition area, has no extrema. Figure 5 shows that the maximum value on the $\mathrm{Y}$-axis is reached at a value of $\mathrm{Y}=500 \mathrm{~mm}$. It is important to note that the gradient of the displacement distribution does not give an unambiguous understanding of the angular position of the milling head, in which the largest values are observed. As a result, a surface scan of the values in polar coordinates $(\chi, \varphi)$ was created (see Fig. 6).
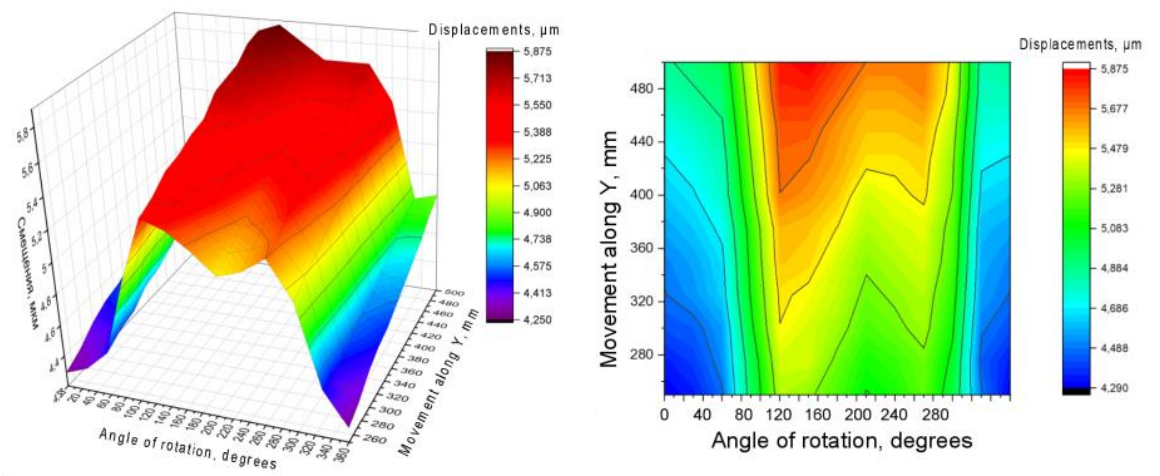

Fig. 5. Surface and plane of values in a solid column.

As the coordinate of the position of the milling head along the $\mathrm{Y}$ axis increases, the resulting displacements increase proportionally. The family of curves $(\chi, \varphi)$ are elliptic curves with a center shifted relative to the A axis. The curves have the same order and are equidistant to each other, i.e. the increment in each angular position when moving along Y is approximately constant. It is impossible to unambiguously visually determine the maximum angular coordinate at which the maximum displacements are observed. In this regard, the function $(\chi, \varphi)$ at $\mathrm{Y}=500$ was approximated by a ninth degree polynomial with the degree of correspondence R2 $=0.99$ (see Fig. 7). To find the extremum, it was customary to use the built-in functions of the OriginPro 2020b mathematical program, since the analytical finding of the extremum of the polynomials is extremely difficult or impossible [14]. As a result, the maximum resulting displacement and the angle at which it occurs were 
found. In a similar way, for the minimum value $\mathrm{Y}=250 \mathrm{~mm}$, the minimum offset value and the angle at which it occurs were found. According to the formula, the corresponding stiffness was determined, the results are presented in table 1.

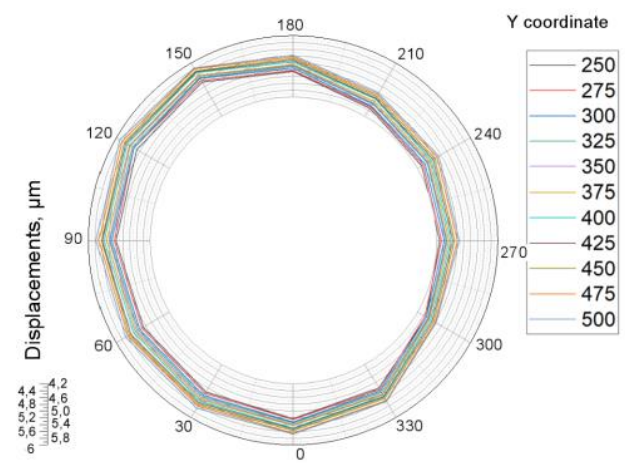

Fig. 6. Dependence of displacements on the angle of rotation of the milling head.

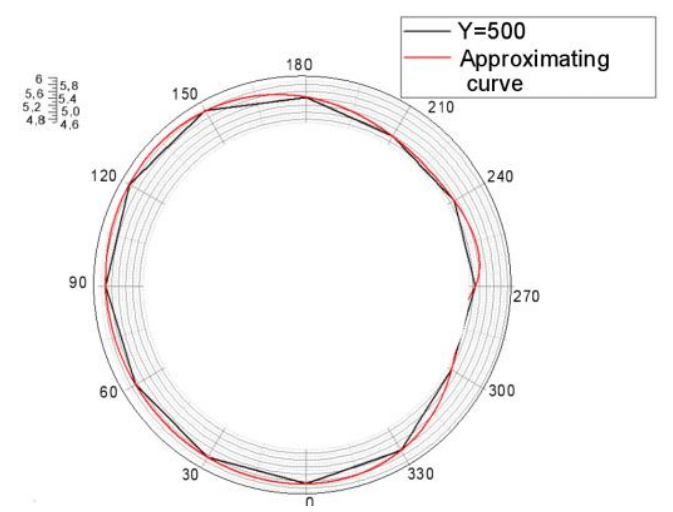

Fig. 7. Maximum displacement at $\mathrm{Y}=500$.

Table 1. Boundary conditions in a solid frame.

\begin{tabular}{|c|c|c|c|}
\hline $\mathrm{Y}, \mathrm{mm}$ & Displacement $\chi, \mu \mathrm{m}$ & Angle of rotation $\varphi$, degree & Rigidity, $\mathrm{H} / \mu \mathrm{m}$ \\
\hline 250 & 4.29 & 120.25 & 1078 \\
\hline 500 & 5.875 & 119.85 & 788 \\
\hline
\end{tabular}

To check the FEM calculations, the data of field experiments on measuring the rigidity of similar box-shaped structures, carried out by the ENIMS Institute of Machine-Tool Building, were used. As a model for comparison, an experiment on the bending of boxshaped vertical frames and revealing the dependence of the arising displacements on the point of application of the force was chosen. The calculated dependence on the modeling parameters (fixing, geometric dimensions) are presented in Fig. 8.

To verify the mathematical model, a study was carried out, similar to a full-scale experiment, the output data of which are the values of the rigidity coefficients $R R^{\prime}$ of the simplest box-shaped rigidity structures.

The root-mean-square deviation of the FEM calculation data $R R^{\prime}$ from the experimental data of ENIMS $R^{\prime}$ is (for three curves of the rigidity coefficients, respectively): 
The studies carried out unambiguously confirm that the modeling method allows obtaining reliable data, since the maximum permissible deviation from a full-scale experiment in mechanical engineering should not exceed $20 \%$.

Since the calculations based on numerical methods are a certain approximation [15], to increase the accuracy of calculations, the values of the minimum possible wall thicknesses were obtained in two ways:

- extrapolation of the surface of the values at a constant angle of rotation of the milling head (in which the maximum displacements are observed);

- extrapolation of the curve at a constant Y coordinate.

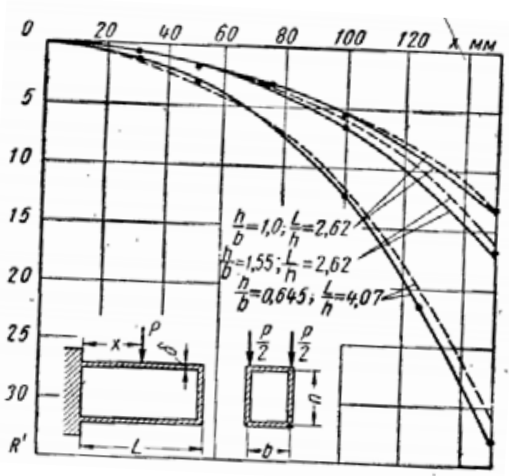

Fig. 8. Experimental dependence of displacements $\left(\mathrm{R}=1 * 10^{3}\right)$.

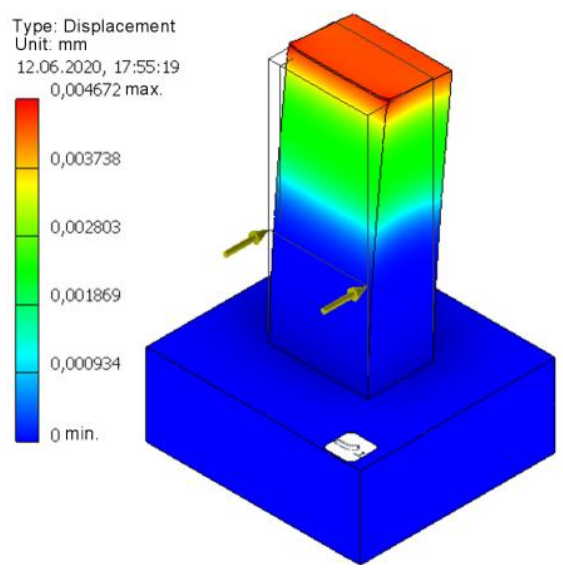

Fig. 9. Calculation of $f$ at $\mathrm{x}=150 \mathrm{~mm}$.

As parameters for the first calculation, the wall thickness $t$ was chosen in the range from the maximum (solid stand with full filling of the material) to the wall thickness $t=15 \mathrm{~mm}$ with a division into 11 intervals, the amount of displacement in the working field $Y=250$ 500 with a step $\mathrm{y}=25 \mathrm{~mm}$ and $\varphi=$ Const $=120$ degrees. The construction of a mathematical model at a fixed angle of rotation of the milling head, while varying the displacement along the $\mathrm{Y}$ axis and the wall thickness $\mathrm{t}$, determines the surface of the displacement values in a system consisting of 121 displacement coordinates. Figure 10 shows a family of curves representing displacements occurring at different wall thicknesses.

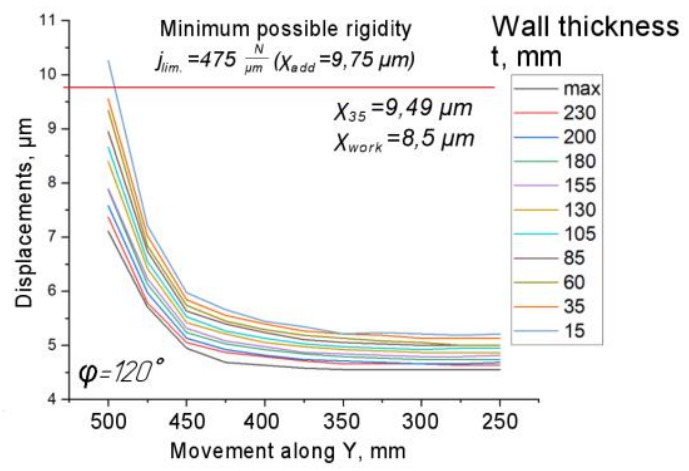

Fig. 10. The result of the calculation at a constant angle of rotation. 
Based on the minimum possible rigidity of the system, a limiting straight line was drawn with the limiting value of the displacements, namely, at $\chi$ add $=9.75 \mu \mathrm{m}$. It is determined that the range of sizes $t=$ max- 35 is included in the range of permissible values, at $\mathrm{t}=15$ - not included. The maximum displacements at wall thickness $\mathrm{t}=35 \mathrm{~mm}$ (original design) are $\chi 35=9.49 \mu \mathrm{m}$. Proceeding from the fact that the permissible value of the wall thickness lies in the range $\mathrm{t}=15-35 \mathrm{~mm}$, an additional study was carried out. Using the same data, the distribution of experimental points in space was constructed (see Fig. 11).

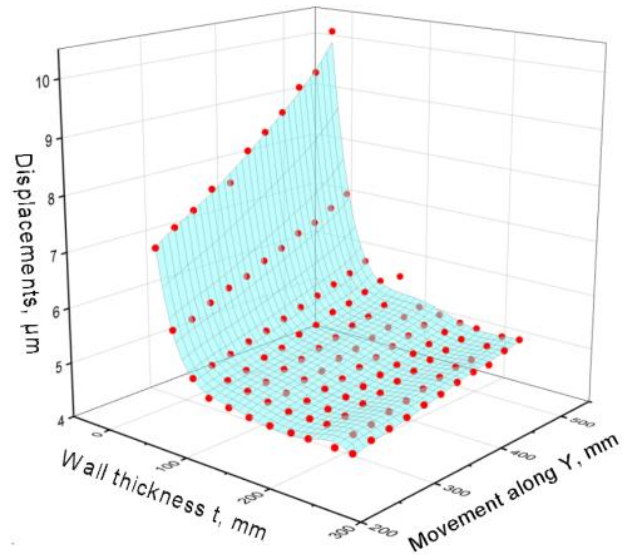

Fig. 11. Distribution of values in the definition area.

Using the built-in function "SurfaceFit" of the OriginPro software, a regression model was built using a polynomial function of the 5th degree along the $t$ and $\mathrm{Y}$ axes. As a result, a function of two variables was obtained, with the coefficient of determination R2 $=0.95$. The resulting function is equal to:

$\chi=-37.1-0.14 \mathrm{Y}+0.63 \mathrm{t}+0.0017 \mathrm{Y}^{2}-0.03 \mathrm{t}^{2}-1.34 \mathrm{Y}^{3} \ldots$ $\ldots-7.2 \mathrm{Y}^{3} \mathrm{t}^{3} \cdot 10^{-13}+6.39 \mathrm{Yt}^{4} \cdot 10^{-14}+8.58 \mathrm{t}^{5} \cdot 10^{-14}$.

Using built-in software, when substituted $\chi \mathrm{add}=9.75 \mu \mathrm{m}$ and $\mathrm{Y}=500 \mathrm{~mm}$ into the resulting function, minimum permissible wall thickness $t_{\min \varphi}=31.2 \mathrm{~mm}$ was found. Based on the experiment, it can be unambiguously asserted that the maximum displacements occur at $\mathrm{L}=\max =500 \mathrm{~mm}$.

Within the framework of the study, a similar modeling was also carried out. However, the angle of rotation of the milling head $\varphi$ in the full working range of $0-360$ degrees with a step of 30 degrees was chosen as the variable parameters, and the wall thickness $t$ was chosen in the range from the maximum (solid stand) to $15 \mathrm{~mm}$, with a division into 11 intervals. As a result of this experiment, 121 displacement values were obtained for various wall thicknesses. The calculation result is presented in graphical form in polar coordinates in Fig. 12.

The simulation results are a family of equidistant closed curves in the milling head range. Obviously, the previously stated hypothesis is unambiguously correct, and the maximum displacement is distributed along the vector passing through 120 degrees. In this field of values, the limit was determined - a red circle (see Fig. 12). After analyzing the data, it was concluded that the permissible displacements lie in the range of $t=$ max-35 $\mathrm{mm}$, and $\mathrm{t}=15 \mathrm{~mm}$ is not included in the range of permissible values. 


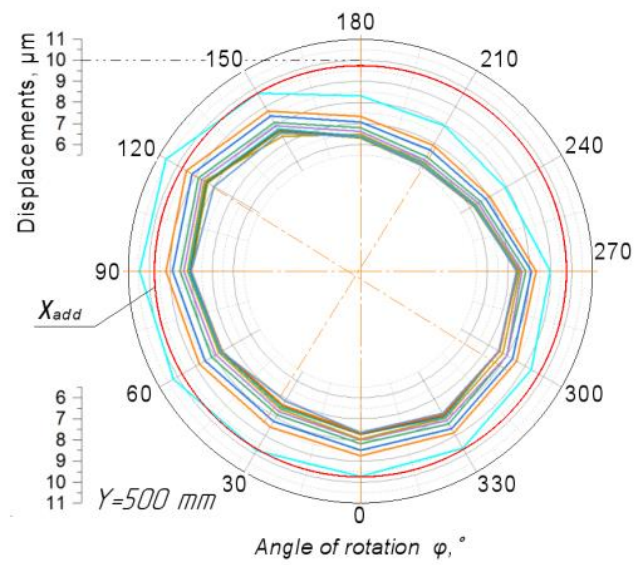

Fig. 12. Calculation with varying the angle of rotation.

Using the assumption that all the resulting curves are equidistant to the previous ones, an extreme curve was found that would have only one point with a circle of permissible values with $\mathrm{r}=\chi$ add.

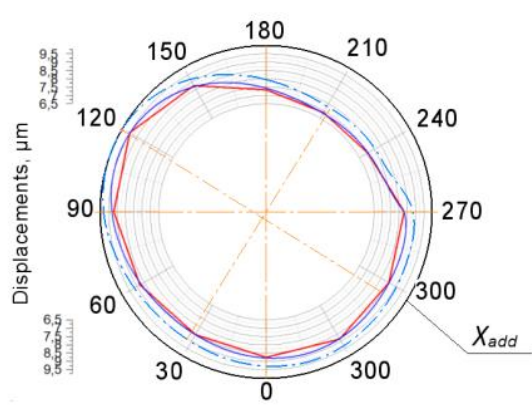

a)

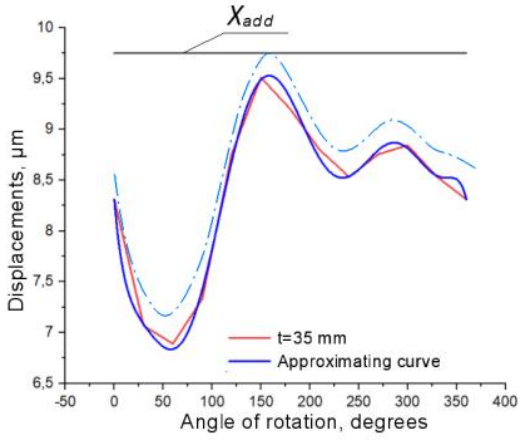

b)

Fig. 13. Limit curve.

Figure 13 denotes the search for the limit value when plotting a graph in polar coordinates (Fig. 13a) and its development drawing on the plane (Fig. 13b).

Using the simplefit and connect/touch functions of the OriginPro software, the equidistant of this curve was found, which will touch the circle with $r=\chi a d d$. Thus, a curve was obtained that corresponds to the wall thickness tminY $=31.5 \mathrm{~mm}$, the maximum displacement of which is $\chi$ add $=9.75 \mu \mathrm{m}$. Based on this, the deformation in the support system was determined in the working position $\varphi=0$ deg at $\mathrm{t}=35 \mathrm{~mm}$, then $\chi 35 \mathrm{r}=8.5 \mu \mathrm{m}$. Thus, the maximum rigidity in the working position with the initial wall thickness will be $\mathrm{j}_{35 r}=\frac{F}{\chi_{35 \mathrm{r}}}=\frac{4627}{8.5}=550 \frac{\mathrm{N}}{\mu \mathrm{m}}$.

\section{Discussion}

As a result of the study, conclusions were drawn that confirm the theoretical prerequisites for a decrease in the total metal consumption of the structure through the use of parametric 
optimization methods to find the smallest wall thickness of the milling column while maintaining the required parameters of the structure rigidity and processing accuracy.

Structurally, it is worth highlighting three main provisions that confirm the proposed hypothesis:

1) The maximum nominal rigidity of the system with the initial wall thickness $t=35$ $\mathrm{mm}$ is $\mathrm{j}_{35}=\frac{F}{\chi_{35}}=\frac{4627}{9.49}=488 \frac{N}{\mu \mathrm{m}}$ over the entire range of movement of the milling head along $\mathrm{Y}=250-500 \mathrm{~mm}$ and around the rotary axis from $\varphi=0 \ldots 360 \mathrm{deg}$. The maximum rigidity in the working position $\varphi=0$ is equal to $\mathrm{j}_{35 \mathrm{p}}=\frac{F}{\chi_{35 \mathrm{p}}}=\frac{4627}{8.5}=550 \frac{\mathrm{N}}{\mu \mathrm{m}}$.

2) The rigidity margin for the maximum displacement of the working body is equal to:

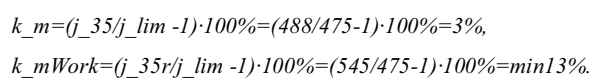

3) The minimum possible wall thickness, while maintaining the specified accuracy, is tminY $=31.5 \mathrm{~mm}$, which makes it possible to reduce the mass of the initial assembly by $50-80 \mathrm{~kg}$ while maintaining the specified accuracy and structural rigidity.

Experimental confirmation of the theory put forward within the framework of this study allows concluding that the use of parametric optimization methods for given objective functions makes it possible to determine the most optimal geometry of the supporting structures of the working elements of metal-cutting equipment, while significantly reducing the required metal consumption of the working bodies and maintaining the required accuracy indicators of the resulting products.

\section{Conclusion}

A multivariate analysis of the machine's supporting structure was carried out. The vertical column of the milling column was checked by means of a finite element calculation for compliance with the occurrence of maximum elastic deflections during the gear hobbing operation. As a result of which conclusions were made about the suitability of work in the range of permissible processing accuracy, the possibility of reducing the material consumption of the structure was identified, the safety factor for rigidity, i.e. for the maximum possible applied force, was determined.

\section{References}

1. R.X. Gao, P. Wang, Procedia CIRP 59, 2-7 (2017)

2. G. He, L. Guo, S. Li, D. Zhang, Advances in Mechanical Engineering 11 (2017)

3. V.T. Portmana, V.S. Chapskya, Y. Shneora, E. Ayalon, CIRP 25th Design Conference Innovative Product Creation 36, 111-116 (2015)

4. J. Fleischer, C. Munzinger, M. Tröndle,. Production Engineering 2, 85-90 (2008)

5. M. McConahaa, V. Venugopal, S. Anan, Procedia Manufacturing 48, 634-642 (2020)

6. Zh. Li, Y. Fu, L. Shi, H. Bian, Advances in Engineering Research 63, 370-376 (2016)

7. M. Law, Y. Altintas, A. Srikantha Phani, International Journal of Machine Tools \& Manufacture 68, 81-90 (2013)

8. N. Kepczak, W. Pawlowski, Iran J Sci Technol Trans Mech. 44, 523-532 (2020)

9. D. Kono, T. Inagaki, A. Matsubara, I. Yamaji, Precision Engineering 3, 650-657 (2013)

10. J. B.Li, G. Hon, Z. Wanga, W. Wu, Y. Chen, Procedia CIRP 3, 626-631 (2012) 
11. K. Szipka, Th. Laspas, A. Archenti, Precision Engineering 51, 59-67 (2018)

12. Th. Laspas, N. Theissen, A. Archenti, Precision Engineering 65, 164-170 (2020)

13. I. Tănase, A. Ghionea, I. Ghionea, Proceedings in Manufacturing Systems 5, 243-248 (2010)

14. M. Zaeh, D. Siedl, CIRP Annals - Manufacturing Technology 56, 383-386 (2007)

15. I.A. Daniyan, I. Tlhabadira, O.O. Daramola, K. Mpofua, Procedia CIRP 84, 861-867 (2019) 